\title{
Body Weight Gain Original Result
}

National Cancer Institute

\section{Source}

National Cancer Institute. Body Weight Gain Original Result. NCI Thesaurus. Code

C119767.

The outcome of the body weight gain assessment as originally received or collected. 